\title{
Editorial: Quantum Information and Quantum Computing for Chemical Systems
}

\author{
Sabre Kais ${ }^{1 *}$, Travis Humble ${ }^{2}$, Karol Kowalski ${ }^{3}$, Ivano Tavernelli ${ }^{4}$, Philip Walther ${ }^{5}$ and \\ Jiangfeng $D u^{6}$
}

${ }^{1}$ Chemistry Department, Purdue University, West Lafayette, IN, United States, ${ }^{2}$ Oak Ridge National Laboratory, Oak Ridge, TN, United States, ${ }^{3}$ Pacific Northwest National Laboratory, Richland, WA, United States, ${ }^{4}$ IBM Quantum, IBM Research - Zürich, Rüschlikon, Switzerland, ${ }^{5}$ Physics Department, University of Vienna, Vienna, Austria, ${ }^{6}$ Physics Department, University of Science and Technology of China, Hefei, China

Keywords: quantum, quantum computing, quantum chemistry, quantum information, quantum science

\section{Editorial on the Research Topic}

Quantum Information and Quantum Computing for Chemical Systems

Quantum computing has emerged as an exciting inter-disciplinary research topic that cuts across the traditional fields of physics, computer science, and engineering [1]. It is a revolutionary model of computation that has offered new insights into methods for modeling and simulation of chemical systems [2]. Applications of quantum computing to chemistry have demonstrated rapid progress on both theoretical and experimental fronts [3]. Past theoretical efforts have shown how to adapt quantum computation to a variety of problems including electronic structure and molecular dynamics. In addition, the development of quantum algorithms for quantum chemistry has been stimulated by the greater availability of more capable quantum computing devices [4]. Advances in the number and quality of qubits continues to enable remarkable proof-of-concept demonstrations working towards a milestone of quantum computational advantage [5].

However, many challenges remain along the way toward practical quantum advantages of

\section{OPEN ACCESS}

Edited and reviewed by: Malgorzata Biczysko, Shanghai University, China

${ }^{*}$ Correspondence:

Sabre Kais

kais@purdue.edu

Received: 05 August 2021 Accepted: 13 August 2021 Published: 01 September 2021

Citation:

Kais S, Humble T, Kowalski K, Tavernelli I, Walther P and Du J (2021)

Editorial: Quantum Information and Quantum Computing for Chemical Systems.

Front. Phys. 9:753618.

doi: 10.3389/fphy.2021.753618 quantum computing for computational chemistry. Several key examples are the development of new quantum algorithms for solving chemical problems thought to be intractable for classical computers, the efficient representation of those models within quantum computers, the verification and validation of chemical simulations using quantum computers, and the testing and evaluation of real-world problems on currently available devices.

The purpose of this research topic Quantum Information and Quantum Computing for Chemical Systems is to present the latest snapshot of leading theoretical concepts, computational methods, and experimental demonstrations of computational chemistry with quantum computers. In the opening article, Yang et al. develop new methods for measuring the time-dependent one-particle probability densities from quantum simulation circuits. They apply these methods to time-dependent density functional theory successfully using numerical simulation of the helium hydride ion with 4- and 8orbital models. Bylaska et al. demonstrate the advantages of an efficient representation of electronic correlations based on a plane-wave basis for electronic structure calculations of molecular hydrogen. The new correlation optimized virtual orbitals are benchmarked against molecular hydrogen and found to be significantly better at capturing correlation in plane-wave calculations. Claudino et al. address the accuracy of variational quantum algorithms for modeling the exact FCI state of small molecular systems. Their numerical results find that VQE and ADAPT-VQE methods offer similar accuracies with significantly different circuit complexities. 
Several contributions address the model of quantum computation and the extension to new paradigms for computation. This includes the contribution from Wang et al. on the use of qudits for quantum computing, which reviews techniques for building algorithms and circuits that apply to available experimental devices before presenting specifications of leading quantum algorithms using these methods. Dixit et al. present on the advantages of quantum annealing for training restricted Boltzmann machines against Markov chain Monte Carlo, which show promise of reducing the number of iterations needed to this manuscript has been authored by UT-Battelle, LLC under Contract No. DE-AC05-00OR22725 with the U.S. Department of Energy. The United States Government retains and the publisher, by accepting the article for publication, acknowledges that the United States Government retains a non-exclusive, paid-up, irrevocable, worldwide license to publish or reproduce the published form of this manuscript, or allow others to do so, for United States Government purposes. The Department of Energy will provide public access to these results of federally sponsored research in accordance with the DOE Public Access Plan (http://energy.gov/downloads/doe-public-accessplan) realize highly accurate classification. Bhatia present a model for chemical reactions using quantum finite automata using the Belousov-Zhabotinsky reaction network as a demonstration.

Applications to chemistry is one of the fastest growing areas in quantum computing and several contributions provide examples of the many different approaches under development. Komarova et al. discuss how parallelization can be used in quantum computing for simulations of vibrational dynamics. They adapt this approach to a platform of semiconducting quantum dot dimers for computing the mean position and momentum of the vibrations in parallel. Cheng et al. detail approaches to applications of quantum computing for biochemical systems focusing on methods that split the computational overhead between conventional and quantum computers. This "divide and conquer" approach offers a promising set of strategies for exploring problems in shell transition-metal and conjugated pielectron strongly correlated systems as well as non-covalent

\section{REFERENCES}

1. National Academies of Sciences. Engineering, and Medicine, Quantum Computing: Progress and Prospects. Washington, DC: National Academies Press (2019).

2. Cao Y, Romero J, Olson JP, Degroote M, Johnson PD, Kieferová M, et al. Quantum Chemistry in the Age of Quantum Computing. Chem Rev (2019) 119(19):10856-915. doi:10.1021/acs.chemrev.8b00803

3. McArdle S, Endo S, Guzik A, Benjamin S, and Yuan X. Quantum Computational Chemistry. Rev Mod Phys (2020) 92(1):015003. doi:10.1103/ revmodphys.92.015003

4. Bauer B, Bravyi S, Motta M, and Chan GK-L. Quantum Algorithms for Quantum Chemistry and Quantum Materials Science. Chem Rev (2020) 120(22):12685-717. doi:10.1021/acs.chemrev.9b00829

5. Arute F, Arya K, Bacon D, Boixo S, Chen Y, Babbush R, et al. Hartree-Fock on a Superconducting Qubit Quantum Computer. Science (2020) 369:1084-9. doi:10.1126/science.abb9811 bonding. Kardashin et al. give a demonstration of quantum machine learning for constructing a tensor network that approximates the eigenvector of a black box model. This new connection forms a bridge between the language of tensor networks and variational quantum algorithms with plans for near-term demonstrations.

This exciting collection of work highlights the ongoing development of quantum computing for chemistry. Our editorial team for this research topic is sincerely grateful to all the reviewers that offered their perspective and expertise in selecting this outstanding collection of contributions. Combining the expertise of the chemical physics community with insights from the quantum computing community continues to lead to a fresh understanding of important chemical processes and the emergence of the novel manybody formulations.

\section{AUTHOR CONTRIBUTIONS}

All authors listed have made a substantial, direct, and intellectual contribution to the work and approved it for publication.

\section{FUNDING}

$\mathrm{TH}$ and KK were supported by the "Embedding Quantum Computing into Many-body Frameworks for Strongly Correlated Molecular and Materials Systems" project, which is funded by the United States Department of Energy, Office of Science, Office of Basic Energy Sciences, the Division of Chemical Sciences, Geosciences, and Biosciences, and by the Quantum Science Center, a National Quantum Information Science Research Center of the U.S. Department of Energy. SK, TSH and KK also acknowledge the support from the Quantum Science Center, a National Quantum Information Science Research Center of the U.S. Department of Energy.

Conflict of Interest: The authors declare that the research was conducted in the absence of any commercial or financial relationships that could be construed as a potential conflict of interest.

Publisher's Note: All claims expressed in this article are solely those of the authors and do not necessarily represent those of their affiliated organizations, or those of the publisher, the editors and the reviewers. Any product that may be evaluated in this article, or claim that may be made by its manufacturer, is not guaranteed or endorsed by the publisher.

Copyright (c) 2021 Kais, Humble, Kowalski, Tavernelli, Walther and Du. This is an open-access article distributed under the terms of the Creative Commons Attribution License (CC BY). The use, distribution or reproduction in other forums is permitted, provided the original author(s) and the copyright owner(s) are credited and that the original publication in this journal is cited, in accordance with accepted academic practice. No use, distribution or reproduction is permitted which does not comply with these terms. 